\title{
A Positron Emission Tomography Study Of Methylphenidate in Adults with ADHD: Alterations in Resting Blood Flow and Predicting Treatment Response
}

\author{
Julie B Schweitzer*,', Douglas O Lee ${ }^{2}$, Russell B Hanford², Malle A Tagamets', John M Hoffman ${ }^{3,4}$, \\ Scott T Grafton ${ }^{5}$ and Clinton D Kilts ${ }^{2}$ \\ 'Maryland Psychiatric Research Center and Division of Child Psychiatry, University of Maryland School of Medicine, USA; ${ }^{2}$ Department of \\ Psychiatry and Behavioral Sciences, Emory University School of Medicine, USA; ${ }^{3}$ Departments of Neurology and Radiology, Emory University \\ School of Medicine, USA; ${ }^{5}$ Center for Cognitive Sciences, Dartmouth College, USA
}

\begin{abstract}
A hallmark symptom of attention-deficit hyperactivity disorder (ADHD) is an excess of motoric behavior or hyperactivity. Methylphenidate $(\mathrm{MPH})$ is known to reduce hyperactivity in individuals with ADHD. Yet little is known about how it alters neural activity and how this relates to its clinical effects. The goal of this study is to examine $\mathrm{MPH}$-induced changes during resting brain metabolism, and to examine how these changes correlate with measures of behavioral response to the drug. Measures of regional cerebral blood flow ( $\mathrm{CCBF}$ ) using positron emission tomography (PET) were acquired at rest for ten adult subjects with ADHD during both an unmedicated state and after a 3-week period of chronic dosing with a clinically optimal dose of $\mathrm{MPH}$. Compared with the on-MPH condition, the off$\mathrm{MPH}$ condition was associated with relative increases in rCBF bilaterally in the precentral gyri, left caudate nucleus, and right claustrum. The on-MPH condition was associated with relative increases in $\mathrm{CCBF}$ in the cerebellar vermis. A correlational analysis measured the relation between $\mathrm{rCBF}$ in the off-medication condition to change in ADHD ratings between the off- and on-MPH condition to identify brain regions associated with treatment response. The degree of change in the ratings was negatively correlated with rCBF increases in the midbrain, cerebellar vermis, and the precentral and middle frontal gyri in the off-MPH condition. The majority of these brain regions are involved in the planning and execution of motor behavior. These data suggest that MPH modulates brain regions associated with motor function to achieve a reduction in ADHD symptoms.

Neuropsychopharmacology (2003) 28, 967-973, advance online publication, 26 March 2003; doi: I 0. I038/sj.npp. 13001 I0
\end{abstract}

Keywords: ADHD; methylphenidate; positron emission tomography; treatment response; cerebellar vermis

\section{INTRODUCTION}

Methylphenidate (MPH) is the drug of choice for the treatment of attention-deficit/hyperactivity disorder (ADHD) (Safer and Malever, 2000). MPH reduces the symptoms of situationally inappropriate and high levels of motor activity, poor sustained attention, and increased impulsivity (see eg Borcherding et al, 1989; Rapport et al, 1985; van der Meere et al, 1995). Although approximately 11 million MPH prescriptions are written per year in the United States (Woodworth, 2000) the mechanisms of MPH

\footnotetext{
*Correspondence: Dr J Schweitzer, Maryland Psychiatric Research Center, White Building, PO Box 21247 , Baltimore, MD 21228, USA, Tel: + I 410402 6027; Fax: + I 410788 3394,

${ }^{4}$ Current address: National Cancer Institute, National Institute of Health, USA

E-mail: jschweit@mprc.umaryland.edu

Received 28 May 2002; revised 08 August 2002; accepted 31 October 2002

Online publication: 06 November 2002 at http://www.acnp.org/ citations/Npp I 10602427
}

effects beyond its molecular actions, and the biological basis of poor response, remain poorly understood. Noninvasive in vivo imaging methods have emerged as a promising tool to explore the nonpharmacological effect of psychotropic drugs on regional brain activity (Salmeron and Stein, 2002).

A review of the literature on stimulants and ADHD suggests that the effectiveness of MPH is owing to its actions on both the dopamine and norepinephrine systems (Solanto, 1998). There is a growing body of research on the effects of MPH on the dopamine system in humans. Therapeutic oral doses of MPH appear to block the function of the dopamine transporter protein (DAT, Volkow et al, 1998) and increase brain extracelluar dopamine levels (Volkow et al, 2001). An imaging positron emission tomography (PET) study using MPH labeled with carbon$11\left({ }^{10} \mathrm{C}\right)$ in normal volunteers showed selective binding of MPH in striatum in humans (Volkow et al, 1995). Most of what is known about the effects of MPH on the norepineperine system is based on nonhuman studies and these show an effect of drug on extracellular concentrations 
of NE (Kuczenski and Segal, 2001), in addition to the wellknown effects on dopamine.

Imaging studies on the effects of MPH on blood flow in children and adults with ADHD implicate dysfunction of the striatum. Several different brain-imaging techniques have shown that MPH increases neuronal activity in the striatum in children and adults treated with MPH (Lou et al, 1984; Teicher et al, 2000; Vaidya et al, 1998).

The frontal cortex is also implicated as a substrate for $\mathrm{ADHD}$ and MPH with accumulating evidence pointing to abnormalities in structural volume (Castellanos et al, 1996) and function (Bush et al, 1999; Rubia et al, 1999; Schweitzer et al, 2000; Vaidya et al, 1998; Zametkin et al, 1990) in this region in subjects with ADHD. Early PET-imaging studies on stimulant administration (acute oral, chronic oral, and intravenous administration) showed a lack of effect on frontal glucose metabolism in adults with ADHD (Ernst et al, 1994, 1997; Matochik et al, 1994). Recent functional magnetic resonance imaging fMRI studies (Vaidya et al, 1998), however, suggest that MPH increases frontal cortical activity in children with ADHD (Teicher et al, 1996).

In addition to interest in striatum and frontal cortex, ADHD researchers are now focusing more attention on the cerebellum. Structural imaging studies in ADHD have repeatedly shown abnormalities in the cerebellum in children with ADHD (Berquin et al, 1998; Castellanos et al, 2001; Mostofsky et al, 1998). A recent fMRI study expanded knowledge of the relation between stimulants and the cerebellum (Anderson et al, 2002). Anderson and colleagues measured the effects of MPH on steady-state blood volume on the cerebellar vermis and demonstrated a dose-dependent effect of the drug in children with ADHD (Anderson et al, 2002). This study revealed that the direction of the effect of MPH on blood flow depends on the basal rate of motor activity of the child.

Little is known about the methods for predicting response to MPH. A review of the studies on predicting positive MPH response suggests that at best, only weak associations exist between either behavioral measures or neurochemistry and drug response (Gray and Kagan, 2000). Functional brain imaging as yet has not been looked to as a method for predicting drug response to $\mathrm{MPH}$, although these techniques have yielded novel insights into the pharmacotherapy of major depression (Mayberg et al, 2000). The current study uses a pharmacological PET (phPET) approach to address the question of how MPH alters rCBF in adults with $\mathrm{ADHD}$ and what patterns of $\mathrm{rCBF}$ alteration correspond to improvement in ADHD symptoms.

The specific goals of the present study were two-fold: (1) To define the anatomy of regional cerebral blood flow (rCBF) responses related to MPH in men with ADHD and (2) to identify resting patterns of $\mathrm{rCBF}$ in unmedicated participants that might predict response to MPH. The study reported here differs from previous PET and most fMRI studies of pharmacological effects (Salmeron and Stein, 2002) in that the rCBF responses were determined at rest, and therefore unconfounded by interactions between a cognitive activation task and drug effect. This resting study is part of a more extensive study that does include cognitive activation tasks, and forms a baseline for the interpretation of subsequent studies that use cognitive activation paradigms to define the effects of MPH on ADHD.

\section{METHODS}

\section{Subjects}

Participants included ten men diagnosed with ADHD, combined type (American Psychiatric Association, 1994). To be diagnosed with ADHD, combined type participants had to meet the DSM-IV criteria: having at least six of nine symptoms of inattention and six of nine symptoms of hyperactivity and/or impulsivity. Furthermore, the symptoms had to have arisen in childhood and persisted over development into adulthood. Four of the ADHD subjects had a prior history of MPH treatment, two subjects as children and two as adults. The subjects medicated as children had been stimulant free for approximately 10 years. One adult had been stimulant free for several months and the other for 8 days before the off-medication images were collected.

A school of medicine-based adult ADHD clinic and local advertisements were used to recruit the participants. The screening process included a thorough review of childhood and adult psychological and educational history, family psychiatric history; completion of the Mini-SCID for DSMIV (First et al, 1996) and the Symptom Checklist-90, Revised (Deragotis, 1986) to screen for non-ADHD psychiatric disorders. Completion of a semi-structured interview based on the DSM-IV criteria for ADHD, the Adult ADHD DSM-IV Rating Scale (Murphy and Barkley, 1996) represented the primary diagnostic instruments used to establish the presence of ADHD. Multiple sources completed the ADHD Rating Scale for DSM-IV including two self-report versions (current behavior and behavior between the ages of 5 and 12); a current observer (eg spouse, close friend); and the mother of the potential participant reviewing behavior between the ages of 5 and 12. The Wechsler Adult Intelligence Scale - 3rd Edition (WAIS-III; Wechsler, 1997) assessed Full Scale intellectual functioning and was combined with the Wide Range Achievement Test - 3rd Edition (WRAT-3; Wilkinson, 1993) to rule out learning disabilities in subjects. The mean $( \pm S D)$ age for the participants was 31.5 (8.2) and the mean ( \pm SD) IQ was $122(10.2)$.

We excluded prospective participants for the following reasons: presence of any clinically significant medical condition, history of brain injury, mental retardation (Full Scale IQ <75), other Axis I disorders, and/or history of nonstimulant pharmacotherapy for greater than 3 weeks. All participants were right-hand dominant (Raczkowski and Kalat, 1974). All subjects had normal neurological histories and exams (DL). Following description of the study and its associated risks, participants gave written informed consent for a protocol approved by the Human Investigations and Radiation Safety Committees of the Emory University School of Medicine. The study was conducted in accordance with the Declaration of Helsinki.

\section{MPH Treatment}

Subjects were started on an MPH dose of $0.5 \mathrm{mg} / \mathrm{kg} / \mathrm{day}$ for the first week, escalating to $0.75 \mathrm{mg} / \mathrm{kg} / \mathrm{day}$ for the second week, and up to $1.0 \mathrm{mg} / \mathrm{kg} /$ day for the third week, unless adverse side effects were noted, until the optimal dose for each subject was found. Dose selection was based on weekly evaluations that included an interview with a review of 
symptoms and side effects by a psychiatrist (DL), completion of the clinical global impression scale (NIMH, 1985) by the psychiatrist (DL), and completion of the side effect scale for psychostimulants (Barkley, 1981) and ADHD rating scale (Murphy and Barkley, 1996) by the subject and a significant other or friend of the subject. Subjects ingested their dose of MPH 60 min before scanning on the day of the imaging at the imaging center. Subjects were administered an average daily dose of $19 \mathrm{mg}( \pm 9.1, S D)$ given as a divided dose (TID). All subjects but one were imaged in the unmedicated condition first and then imaged a second time after achieving optimal behavioral changes on the MPH. One subject was imaged in the medication condition first and then in the unmedicated condition. A review of the data from this one subject does not suggest that the results from this subject differ from the other subjects.

\section{Study Conditions}

During PET scanning subjects were instructed to relax and close their eyes during the acquisition of two $\left[{ }^{15} \mathrm{O}\right] \mathrm{H}_{2}$ resting PET scans of 90 -s duration and spaced 60 min apart. Each subject also performed two different cognitive tasks in separate scans, which are not reported here. Data from the activation tasks will be the subject of a separate report.

A Siemens ECAT 951 PET scanner (Knoxville, TN) acquired images under dim ambient lighting following the bolus intravenous administration of $45 \mathrm{mCi}$ of $\left[{ }^{15} \mathrm{O} \mathrm{H}_{2}\right.$ The scanner collects 31 contiguous $3.375-\mathrm{mm}$-thick slices with an intrinsic resolution of $6 \mathrm{~mm}$ at the center of the field of view. Images were collected parallel to the canthomeatal line. Head movement was minimized by use of a thermoplastic face mask affixed to a customized head holder (TruScan restraint system ${ }^{\mathbb{R}}$ ).

\section{PET Imaging and Data Analysis}

PET images were reconstructed using a measured attenuation correction. The PET images were analyzed using statistical parametric mapping 99 (Wellcome Department of Cognitive Neurology, 1999). Scans from each subject were realigned to each subject's first scan to control for head movement. The PET scans for each condition were averaged, spatially normalized, normalized for global blood flow by proportional scaling, and coregistered to a population representative PET cerebral blood flow atlas centered in Talairach coordinates (Talairach and Tournoux, 1988). PET images were smoothed to a final isotropic resolution of $10 \mathrm{~mm}$ FWHM. Smoothed PET images were normalized to each other by proportional scaling of global activity. A within-subject model was used to calculate the comparisons between the averaged unmedicated from the medicated images using a subtraction analysis. In a separate analysis, the difference scores between unmedicated and medicated self-ratings of ADHD symptoms were correlated with whole-brain $\mathrm{rCBF}$ volumes from the unmedicated PET scans. This yields a map of regions that predict expected response to the medication. SPM whole-volume contrasts were thresholded at a voxel level of $p<0.001$ uncorrected. Significant sites of activation were then defined as contiguous clusters at a threshold of $p<0.05$, corrected for multiple comparisons. Only clusters meeting these criteria are reported in Tables 1 and 2.

\section{RESULTS}

\section{Behavioral Data}

According to self ratings and ratings by spouses and significant others, subjects improved significantly on the ADHD DSM-IV rating scale between the off-medication and on-medication conditions. Group-averaged self-ratings of ADHD symptoms decreased by 25.8 points (scale ranged from 0 to $54 ; t=9.68, p<0.0001$, one-tailed) and ratings by others decreased by 19.5 points (scale ranged from 0 to 54 ; $t=4.42, p<0.002)$. Ratings on the CGI decreased significantly from the off-medication condition (mean of 4.40;

Table I Changes in $\mathrm{rCBF}^{\mathrm{a}}$ Related to Chronic Methylphenidate Administration in Men with ADHD

\begin{tabular}{|c|c|c|c|c|}
\hline \multirow[b]{2}{*}{ Anatomical region } & \multicolumn{2}{|c|}{ Site of activation ${ }^{b}$} & \multirow[b]{2}{*}{$\mathbf{z}$} & \multirow[b]{2}{*}{ Volume } \\
\hline & BA & $\begin{array}{c}\text { Coordinates } \\
(x, y, z)\end{array}$ & & \\
\hline \multicolumn{5}{|l|}{ Unmedicated-medicated condition } \\
\hline Left precentral gyrus & 6 & $-46,4,8$ & 4.91 & |448* \\
\hline Right precentral gyrus & 4 & $44,-10,54$ & 4.45 & 276 \\
\hline Left precentral gyrus & 4 & $-40,-14,50$ & 4.25 & 213 \\
\hline Left caudate nucleus & & $-8,10,14$ & 4.07 & 640 \\
\hline Right claustrum & & $42,4,0$ & 3.74 & 441 \\
\hline \multicolumn{5}{|l|}{ Medicated-unmedicated condition } \\
\hline Cerebellum, posterior (VII/VIIII) & & $-6,-68,-30$ & 4.96 & I587** \\
\hline \multicolumn{5}{|c|}{$\begin{array}{l}\text { arCBF indicates regional cerebral blood flow. } \\
\text { bValues represent the stereotaxic location of voxel maxima or minima with coordinates in Talairach space (Talairach } \\
\text { and Tournoux, 1988) in millimeters lateral to midline }(x) \text {, anterior/posterior to the anterior commissure }(y) \text {, and } \\
\text { superior/inferior to the commissural line }(z) \text {. } \\
\text { "SPM whole volume contrasts were thresholded at a voxel level of } p<0.00 \text { I uncorrected. } \\
\text { Significant sites of activation were then defined as contiguous clusters at a threshold of } p<0.05 \text {, corrected for } \\
\text { multiple comparisons. } \\
\text { *Intensity of voxels significant at } p<0.05 \text { after correcting for multiple comparisons. }\end{array}$} \\
\hline
\end{tabular}


Table 2 Negative Correlations between Changes in $\mathrm{rCBF}^{\mathrm{a}}$ and ADHD Ratings

\begin{tabular}{lcccc}
\hline & \multicolumn{2}{c}{ Site of activation $^{\mathbf{b}}$} & & \\
\cline { 2 - 3 } Anatomical region & BA & $\begin{array}{c}\text { Coordinates } \\
\mathbf{( x , y , ~ z )}\end{array}$ & & Volume $^{\mathbf{c}}$ \\
\hline Midbrain & & $-2,-8,-12$ & 5.84 & $3213^{*}$ \\
Cerebelllum, posterior (Crus II) & & $4,-86,-26$ & 5.65 & $600^{*}$ \\
Left middle frontal gyrus & 9 & $-24,6,40$ & 4.27 & 210 \\
\hline
\end{tabular}

${ }^{a} \mathrm{rCBF}$ indicates regional cerebral blood flow.

b Values represent the stereotaxic location of voxel maxima or minima with coordinates in Talairach space (Talairach and Tournoux, 1988) in millimeters lateral to midline $(x)$, anterior/posterior to the anterior commissure $(y)$, and superior/inferior to the commissural line $(z)$.

'SPM whole volume contrasts were thresholded at a voxel level of $p<0.00$ I uncorrected.

Significant sites of activation were then defined as contiguous clusters at a threshold of $p<0.05$, corrected for multiple comparisons.

*Intensity of voxels significant at $p<0.05$ after correcting for multiple comparisons.

$\mathrm{SD}=0.70)$ to the on-medication condition (mean of 1.90 ; $\mathrm{SD}=0.57 ; t=9.30, \mathrm{df}=19, p=0.0001)$. The side effect scale revealed minimal side effects during the on-medication condition.

\section{PET Data (Subtraction Analysis): Changes in rCBF because of $\mathrm{MPH}$}

Compared with the off-medication condition, MPH treatment was associated with increases in $\mathrm{rCBF}$ in the posterior cerebellum (lobules VII/VIII). The greatest increases were noted in the vermis and a site lateral to the vermis in the right cerebellar hemisphere (see Table 1 and Figure 1). The effect of the MPH was strikingly localized to deep brain



Figure I Relative rCBF increases in on-MPH vs off-MPH condition in 10 men with ADHD. For this figure as well as Figures 2 and 3, the sites of activation displayed are significant at a voxel level of $p<0.00$ I uncorrected and thresholded at $p<0.05$, corrected for multiple comparisons at the cluster level. The number beneath the image indicates the $\mathrm{mm}$ above or below the bicommisural plane. The image illustrates the significant activation in the cerebellum.

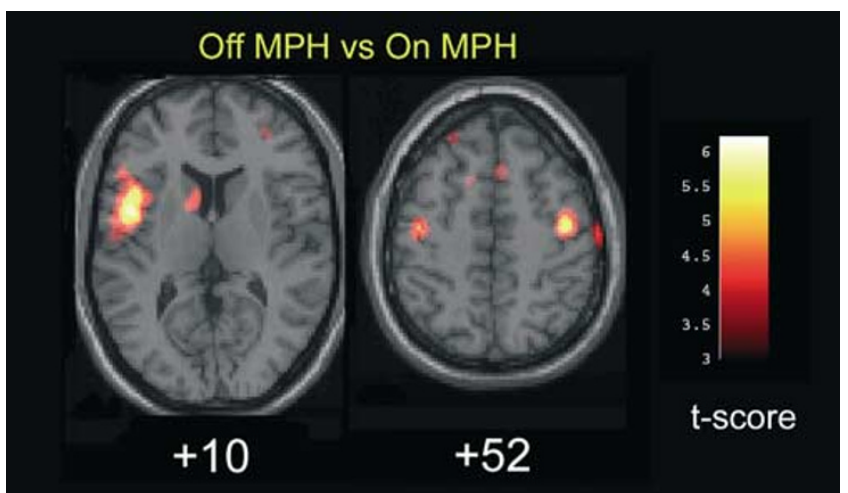

Figure 2 Relative $\mathrm{CCBF}$ increases in off-MPH vs on-MPH condition in 10 men with ADHD. The image illustrates the significant activation in the unmedicated condition bilaterally in the precentral gyrus, left caudate nucleus, and the right claustrum.

structures. Compared with the on-medication condition, the off-medication condition was associated with widespread increases in $\mathrm{rCBF}$ throughout the brain. The off-medication condition increases in $\mathrm{rCBF}$ were in regions superior to those seen in the on-medication condition, including the bilateral precentral gyrus (BA 4), the left caudate nucleus, and the right claustrum (see Figure 2).

\section{PET Data (Correlation Analysis): MPH-Related Improvement on ADHD Ratings and $\mathrm{rCBF}$}

Subjects with greater improvement in ADHD symptom ratings from the unmedicated to the medicated condition showed lower resting $\mathrm{rCBF}$ in the off-medication condition in the midbrain, posterior cerebellum (vermis-Crus II), and left middle frontal gyrus (BA 9). These negative correlations are presented in Table 2 and Figure 3 . The activation at the left precentral gyrus was significant at $p<0.05$ after correcting for multiple comparisons for intensity of voxels. There were no significant positive correlations between $\mathrm{MPH}$-related changes in the ADHD symptom ratings and rCBF.

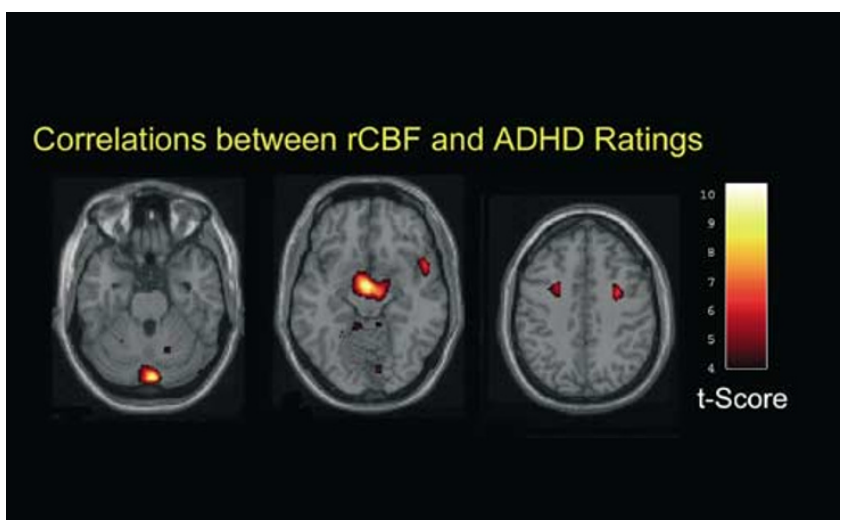

Figure 3 Location of brain activity negatively correlated with change in the ADHD rating scale from the off-MPH to on-MPH condition and $\mathrm{rCBF}$ activity in the off-MPH condition. 


\section{DISCUSSION}

These data identify the anatomical targets of MPH's effect on ADHD and support theories of ADHD based on dysfunctional frontal-striatal circuitry that is influenced by cerebellar and midbrain function (Giedd et al, 2001). A consistent finding in our data is that chronic MPH administration increases $\mathrm{rCBF}$ in the cerebellar vermis, suggesting that it may specifically target this structure. Volkow et al's (1997) finding that MPH consistently increased glucose metabolism in the cerebellum of normal control adult participants suggests that MPH targets this structure in healthy individuals as well. Additional support for the contention that MPH treats ADHD symptoms through the cerebellum was shown in a recent study by Anderson et al (2002) who demonstrated using fMRI that MPH decreases blood volume in the cerebellar vermis in ADHD children with greater symptoms of hyperactivity. Structural data also suggest that the cerebellar vermis may be implicated in ADHD with findings suggesting that the vermis is smaller in children with ADHD compared with healthy children (Berquin et al, 1998).

$\mathrm{MPH}$ is an inhibitor of the norepinephrine and serotonin transporters, in addition to the dopamine transporter. The human cerebellum receives a rich noradrenergic input (Powers et al, 1989) and MPH has been shown to enhance markedly extracellular concentrations of norepinephrine in noradrenergically innervated structures (Kuczenski and Segal, 2001). Therefore, the observed effect of MPH may reflect MPH effects on cerebellar noradrenergic systems, although we cannot rule out contributions owing to effects of MPH on serotonergic systems. The dopaminergic innervation of the human cerebellar vermis is variably described as being sparse to rich (see Melchitzsky and Lewis, 2000), although Volkow et al (2001) have shown using PET and $\left[{ }^{11} \mathrm{C}\right]$ raclopride that MPH does not appear to increase cerebellar dopamine levels. Therefore, it is uncertain whether the effects of MPH on $\mathrm{rCBF}$ in the cerebellar vermis are related to effects on dopaminergic systems. These data are consistent with the hypothesis (Arnsten, 2000; Biederman and Spencer, 1999) that ADHD may be because of dysregulation of the noradrenergic system.

MPH-related changes also include a decrease in the rCBF in brain areas related to motor preparation and action including the precentral gyrus and caudate nucleus. Hyperactivity of these regions could be associated with the excessive motoric activity and poor response inhibition characteristic of ADHD. Decreasing brain activity in these regions may enable individuals with ADHD to engage putative brain regions involved in higher-order functions (ie language) to organize behavior without competition from goal-inappropriate behavioral hyperactivity. The effect of psychostimulants on caudate activity is relatively consistent among previous studies (Matochik et al, 1993; Teicher et al, 2000; Vaidya et al, 1998) showing that right caudate activity is increased by $\mathrm{MPH}$ administration. Our study suggests that the baseline activity in the left caudate may be relatively increased in ADHD subjects.

Our findings suggest that those ADHD subjects with higher off-medication activity of the midbrain, posterior cerebellum, and middle frontal gyrus will be less likely to respond to MPH on indices measured by current ADHD rating scales. Measures of [18F]fluorodopa (FDOPA) activity in children with ADHD suggest baseline dysfunction of the midbrain with abnormal dopamine synthesis or storage of FDOPA activity in the midbrain (Ernst et al, 1999). This neural response to MPH administration may reflect a neural correlate of treatment nonresponse. It is also plausible that those subjects who are naturally compensating for their deficits by increasing brain activity in these regions already will benefit less from the medication because the compensatory activity has already maximized the amount of activity that is possible in those regions. In essence, a ceiling effect is met and the MPH cannot increase the activity any higher in those regions. Indeed, we have other data (JS, unpublished) that show ADHD adults have a higher baseline rate of brain activity in similar regions that we found to correlate with the least amount of change with MPH during performance of a working memory task. The potential presence of 'compensatory' activity requires further exploration in other ADHD samples. The lack of significant positive correlations between $\mathrm{rCBF}$ and changes in symptoms with MPH may reflect a lack of power from this sample size.

The findings of this small study require replication in a larger study sample. This study should also be replicated in children, who are the bulk of the recipients of MPH prescriptions, using less invasive techniques, such as functional magnetic resonance imaging. Future studies will be strengthened by the use of a reversal, double-blind crossover study in which the effects of MPH on rCBF can be discriminated from changes that may be because of alterations in the symptoms related to the chronic dosing condition. This study suggests one method that may be useful in identifying subject characteristics' corresponding to responsivity to MPH. Another potential technique for assessing responsivity to stimulants could include both responders and nonresponders to a medication and correlations between brain activity and measures of the degree of responsivity.

A primary goal of this study was to identify $\mathrm{rCBF}$ response to MPH at rest to aid the interpretation of future studies that use cognitive paradigms to test the substrates of ADHD in response to medication. Our findings provide a guide to interpret data for future ADHD pharmacological studies of MPH. A further goal of the study was to evaluate the use of functional imaging in predicting response to MPH. The results from this study suggest that functional imaging has potential as a tool for identifying individuals' response to pharmacological treatment.

\section{ACKNOWLEDGMENTS}

The study was supported in part by the National Institute of Mental Health (K08 MH01053 \& RO3 MH55550) and the William K Warren Foundation. We thank Delecia Votaw, BS, CNMT, Michael White, BS, CNMT, and Margie Jones, BS, CNMT of the Emory Center for PET, the participants and their spouses, friends, and family members for completion of rating scales. We also thank Tim Ely and Caroline Zink for technical assistance and Henry $\mathrm{H}$ 
Holcomb, MD, Deb Medoff, PhD, and Carol Tamminga, MD for their insightful discussions and support.

\section{REFERENCES}

American Psychiatric Association (1994). Diagnostic and Statistical Manual of Mental Disorders. American Psychiatric Association: Washington, DC.

Anderson CM, Polcari A, Lowen SB, Renshaw PF, Teicher $\mathrm{MH}$ (2002). Effects of methylphenidate on functional magnetic resonance relaxometry of the cerebellar vermis in boys with ADHD. Am J Psychiatry 159: 1322-1328.

Arnsten AF (2000). Genetics of childhood disorders: XVIII. ADHF, Part 2: norepinephrine has a critical modulatory influence on prefrontal cortical function. 39: 1201-1203.

Barkley RA (1981). Hyperactive Children: A Handbook for Diagnosis and Treatment. Guilford Press: New York.

Berquin PC, Giedd JN, Jacobsen LK, Hamburger SD, Krain AL, Rapoport JL (1998). Cerebellum in attention-deficit hyperactivity disorder: a morphometric MRI study. Neurology 50: 1087-1093.

Biederman J, Spencer T (1999). Attention-deficit/hyperactivity disorder (ADHD) as a noradrenergic disorder. Biol Psychiatry 46: 1234-1242.

Borcherding BG, Keysor CS, Cooper TB, Rapoport JL (1989). Differential effects of methylphenidate and dextroamphetamine on the motor activity level of hyperactive children. Neuropsychopharmacology 2: 255-263.

Bush G, Frazier JA, Rauch SL, Seidman LJ, Whalen PJ, Jenike MA (1999). Anterior cingulate cortex dysfunction in attentiondeficit/hyperactivity disorder revealed by fMRI and the counting stroop. Biol Psychiatry 45: 1542-1552.

Castellanos FX, Giedd JN, Berquin PC, Walter JM, Sharp W, Tran T (2001). Quantitative brain magnetic resonance imaging in girls with attention-deficit/hyperactivity disorder. Arch Gen Psychiatry 58: 289-295.

Castellanos F, Giedd J, Marsh W, Hamburger S, Vaituzis A, Dickstein D (1996). Quantitative brain magnetic resonance imaging in attention-deficit hyperactivity disorder. Arch Gen Psychiatry 53: 607-616.

Deragotis LR (1986). Symptom Checklist-90-R: Administration, Scoring, and Procedures Manual, 3rd edn. National Computer Systems, Inc.: Minneapolis.

Ernst M, Zametkin AJ, Matochik JA, Liebenauer L, Fitzgerald GA, Cohen RM (1994). Effects of intravenous dextroamphetamine on brain metabolism in adults with attention-deficit hyperactivity disorder (ADHD). Preliminary findings. Psychopharmacol Bull 30: $219-225$.

Ernst M, Zametkin AJ, Matochik JA, Pascualvaca D, Jons PH, Cohen RM (1999). High midbrain [18F]DOPA accumulation in children with attention deficit hyperactivity disorder. Am J Psychiatry 156: 1209-1215.

Ernst M, Zametkin AJ, Matochik J, Schmidt M, Jons PH, Liebenauer LL (1997). Intravenous dextroamphetamine and brain glucose metabolism. Neuropsychopharmacology 17: 391401.

First M, Gibbon WJ, Spitzer RL (1996). SCID Screen PQ. MultiHealth Systems, North Tonawanda, NY.

Giedd JN, Blumenthal J, Molloy E, Castellanos FX (2001). Brain imaging of attention deficit/hyperactivity disorder. Ann NY Acad Sci 931: 33-49.

Gray JR, Kagan J (2000). The challenge of predicting which children with attention deficit-hyperactivity disorder will respond positively to methylphenidate. J Appl Dev 21: 471-489.

Kuczenski R, Segal DS (2001). Locomotor effects of acute and repeated threshold doses of amphetamine and methylphenidate: relative roles of dopamine and norepinephrine. J Pharmacol Exp Ther 296: 876-883.

Lou H, Henriksen L, Bruhn P (1984). Focal cerebral hypoperfusion in children with dysphasia and/or attention deficit disorder. Arch Neurol 41: 825-829.

Matochik J, Liebenauer L, King C, Szymanski H, Cohen R, Zametkin A (1994). Cerebral glucose metabolism in adults with attention deficit hyperactivity disorder after chronic stimulant treatment. Am J Psychiatry 151: 658-664.

Matochik JA, Nordahl TE, Gross M, Semple WE, King AC, Cohen RM (1993). Effects of acute stimulant medication on cerebral metabolism in adults with hyperactivity. Neuropsychopharmacology 8: 377-386.

Mayberg HS, Brannan SK, Tekell JL, Silva JA, Mahurin RK, McGinnis S (2000). Regional metabolic effects of fluoxetine in major depression: serial changes and relationship to clinical response. Biol Psychiatry 48: 830-843.

Melchitzsky D, Lewis D (2000). Tyrosine hydrolase- and dopamine transporter-immunoractive axons in the primate cerebellum: evidence for a lobular- and laminar-specific dopamine innervation. Neuropsychopharmacology 22: 466-472.

Mostofsky SH, Reiss AL, Lockhart P, Denckla MB (1998). Evaluation of cerebellar size in attention-deficit hyperactivity disorder. I Child Neurol 13: 434-439.

Murphy K, Barkley R (1996). Prevalence of DSM-IV ADHD symptoms in an adult community sample of licensed drivers. $J$ Attention Dis 1: 147-161.

NIMH (1985). Clinical global impression scale. Psychopharmacol Bull 21: 839-844.

Powers RE, O'Connor D, Price D (1989). Noradrenergic systems in human cerebellum. Brain Res 481: 94-199.

Raczkowski D, Kalat J (1974). Reliability and validity of some handedness questionnaire items. Neuropsychologia 12: 43-47.

Rapport MD, DuPaul GJ, Stoner G, Birmingham BK, Masse G (1985). Attention deficit disorder with hyperactivity: differential effects of methylphenidate on impulsivity. Pediatrics 76: 938943.

Rubia K, Overmeyer S, Taylor E, Brammer M, Williams SC, Simmons A (1999). Hypofrontality in attention deficit hyperactivity disorder during higher-order motor control: a study with functional MRI. Am J Psychiatry 156: 891-896.

Safer DJ, Malever M (2000). Stimulant treatment in Maryland public schools. Pediatrics 106: 533-539.

Salmeron BJ, Stein EA (2002). Pharmacological applications of magnetic resonance imaging. Psychopharmacol Bull 36: 102129.

Schweitzer JB, Faber TL, Grafton ST, Tune LE, Hoffman JM, Kilts CD (2000). Alterations in the functional anatomy of working memory in adult attention deficit hyperactivity disorder. $A m J$ Psychiatry 157: 278-280.

Solanto MV (1998). Neuropsychopharmacological mechanisms of stimulant drug action in attention-deficit hyperactivity disorder: a review and integration. Behav Brain Res 94: 127-152.

Talairach J, Tournoux P (1988). Co-Planar Stereotaxic Atlas of the Human Brain. Thieme: New York.

Teicher MH, Polcari A, Anderson CM (1996). Dose dependent effects of methylphenidate on activity, attention, and magnetic resonance measures in children with ADHD. Soc Neurosci Abs 22: 1191.

Teicher MH, Anderson CM, Polcari A, Glod CA, Maas LC, Renshaw PF (2000). Functional deficits in basal ganglia of children with attention- deficit/hyperactivity disorder shown with functional magnetic resonance imaging relaxometry. Nat Med 6 : 470-473.

Vaidya CJ, Austin G, Kirkorian G, Ridlehuber HW, Desmond JE, Glover GH (1998). Selective effects of methylphenidate in attention deficit hyperactivity disorder: a functional magnetic resonance study. Proc Natl Acad Sci USA 95: 14494-14499. 
van der Meere J, Shalev R, Borger N, Gross-Tsur V (1995). Sustained attention, activation and MPH in ADHD: a research note. J Child Psychol Psychiatry 36: 697-703.

Volkow ND, Ding YS, Fowler JS, Wang GJ, Logan J, Gatley JS (1995). Is methylphenidate like cocaine? Studies on their pharmacokinetics and distribution in the human brain. Arch Gen Psychiatry 52: 456-463.

Volkow ND, Wang GJ, Fowler JS, Gatley SJ, Logan J, Ding YS (1998). Dopamine transporter occupancies in the human brain induced by therapeutic doses of oral methylphenidate. Am J Psychiatry 155: 1325-1331.

Volkow ND, Wang GJ, Fowler JS, Logan J, Angrist B, Hitzemann R (1997). Effects of methylphenidate on regional brain glucose metabolism in humans: relationship to dopamine D2 receptors. Am J Psychiatry 154: 50-55.

Volkow ND, Wang G, Fowler JS, Logan J, Gerasimov M, Maynard L (2001). Therapeutic doses of oral methylphenidate significantly increase extracellular dopamine in the human brain. $J$ Neurosci 21: 9414-9418.

Wechsler D (1997). Wechsler Adult Intelligence Scale, 3rd edn. Psychological Corp: New York.

Wellcome Department of Cognitive Neurology (1999). SPM99, Wellcome Department of Cognitive Neurology http: //www. fil.ion.ucl.uk/spm.

Wilkinson G (1993). The Wide Range Achievement Test, 3rd edn. Wide Range, Inc.: Wilmington, DE.

Woodworth T (2000). Amphetamine and methylphenidate; DEA congressional testimony. Committee on Education and the Workforce, Subcommittee on Early Childhood, Youth and Families.

Zametkin A, Nordahl T, Gross M, King A, Semple W, Rumsey J (1990). Cerebral glucose metabolism in adults with hyperactivity of childhood onset. $N$ Engl J Med 323: 1361-1366. 